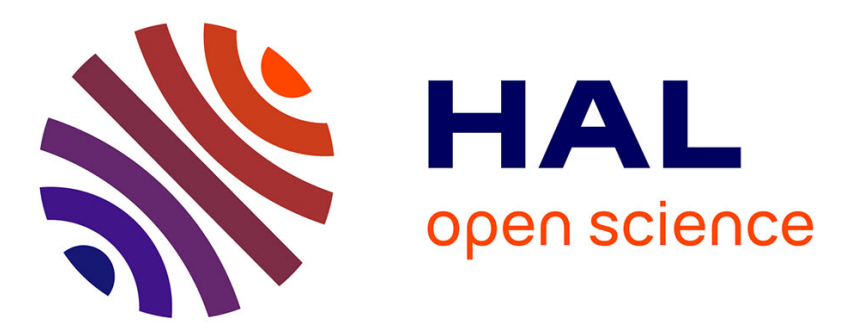

\title{
Assessment of the cervical stiffness in pregnant women using Shear Wave Elastography: a feasibility study
} M Muller, D Ait-Belkacem, Jean Luc Gennisson, M Fink, M Tanter, M Hessabi, D Cabrol, V Tsatsaris

\section{- To cite this version:}

M Muller, D Ait-Belkacem, Jean Luc Gennisson, M Fink, M Tanter, et al.. Assessment of the cervical stiffness in pregnant women using Shear Wave Elastography: a feasibility study. IEEE International Ultrasonics Symposium (IUS), Jul 2013, Prague, Czech Republic. 10.1109/ULTSYM.2013.0002 . hal-03275144

\section{HAL Id: hal-03275144 \\ https://hal.science/hal-03275144}

Submitted on 30 Jun 2021

HAL is a multi-disciplinary open access archive for the deposit and dissemination of scientific research documents, whether they are published or not. The documents may come from teaching and research institutions in France or abroad, or from public or private research centers.
L'archive ouverte pluridisciplinaire HAL, est destinée au dépôt et à la diffusion de documents scientifiques de niveau recherche, publiés ou non, émanant des établissements d'enseignement et de recherche français ou étrangers, des laboratoires publics ou privés. 


\section{Assessment of the cervical stiffness in pregnant women using Shear Wave Elastography: a feasibility study}

\author{
M. Muller, D. Ait-Belkacem, J.L. Gennisson, M. Fink, \\ M. Tanter \\ Insitut Langevin, ESPCI ParisTech, CNRS, INSERM, \\ Université Paris Diderot \\ Paris, France
}

\author{
M. Hessabi, D. Cabrol, V. Tsatsaris \\ Maternité Port-Royal, Hôpital Cochin, AP-HP, Université \\ Paris Descartes \\ Paris, France
}

\begin{abstract}
The quantitative and objective assessment of cervical stiffness has great potential for the estimation of pre-term delivery risk, as well as for the prediction of the success of labor induction. Various methods can be used in vivo for cervical assessment such as vaginal digital examination or static elastography but to our knowledge, none of them can provide a quantitative, absolute and independent evaluation of cervical stiffness in vivo. In this study, such values were obtained in pregnant patients in vivo by using Supersonic shear Imaging technique (SSI). The stiffness of the lower anterior part of the cervix was quantified over a $\varnothing 8 \mathrm{~mm}$ region of interest, during vaginal ultrasound examination in 163 pregnant women. We were able i) to assess the range of normal elasticity values throughout the pregnancy, ii) to demonstrate the intra and interoperator reproducibility of the measurement and iii) to evaluate the potential of SSI for the discrimination of pre-term labour. The elastic modulus of the cervix was found to decrease significantly throughout the pregnancy. This study provides for the first time a database for absolute elastic modulus values of the cervix throughout the pregnancy. Stiffness was observed to decrease with gestional age, which is consistent with results previously obtained using a cervicotonometer. Cervical stiffness is reduced in patients diagnosed with pre-term labour.
\end{abstract}

Keywords: shear wave elastography, supersonic shear imaging, in vivo cervix elasticity, preterm labour

\section{INTRODUCTION}

Spontaneous preterm birth is the leading cause of neonatal mortality. Over one million of babies die every year from complications of pre-term birth, according to the White Paper "Global and Regional Toll of Preterm Birth". Premature neonates who survive have a significantly higher risk of developing serious complications such as cardiovascular and respiratory dysfunction, motor and intellectual deficiencies. Pre-term labour occurs between 22 and 36 weeks of gestational age (GA), and is defined by the association of uterine contractions and cervical modifications. Such symptoms often lead to the hospitalization of patients even though the actual risk of pre-term delivery is not very well determined, probably because of the lack of a reliable diagnostic tool.. The cervical modifications are shortening, dilation and softening of the cervix. They are currently diagnosed clinically through a digital vaginal examination resulting in the determination of an index: the Bishop score. The Bishop score provides satisfying results in the most extreme cases (severe pre-term labour or normal patients) but not in the average pre-term labour cases, mainly because digital vaginal examination leads to a subjective and qualitative assessment of the cervix, when objective and quantitative information would be required. It has been demonstrated that digital examination does not provide accurate diagnosis of true pre-term labor [1]. The quantitative assessment of the cervical length and dilation through ultrasonography improves the diagnosis quality and is now recommended in clinical practice. However, classical ultrasonography only provides morphological information, not related to the mechanical properties of the cervix. Cervical softening, not accessible via classical ultrasonography is known to be the most relevant of the three cervical modifications (shortening, dilation and softening), for pre-term labour diagnosis. Some attempts have been made for the objective quantification of cervical stiffness. A « cervicotonometer " measuring the cervical distensibility index (CDI) was developed in the 1990's. The CDI proved to be a good prognostic indicator in premature labor. It was significantly elevated at the time of hospitalization in women who had a preterm delivery when compared to those who delivered at or after 37 weeks of amenorrhea, whereas clinical scores showed no difference between these two groups [2]. The cervicotonometer required to be inserted in the cervical canal, was therefore considered invasive and is not used for this reason. However, these results demonstrated the relevance of cervical stiffness as a diagnostic parameter for preterm labor.

Various elastography techniques for the measurement of tissue elasticity are available. In static elastography, based on strain imaging, a relative elasticity is retrieved by the analysis of a little compression of the medium induced by the operator. This technique has been used in the cervix in some studies, measuring the rate-of-change in tissue displacement of the cervix in pregnant patients [3]. In Molina's study the authors concluded that the measured elasticity may be influenced by the force applied on tissue by the transducers. Parra-Saavedra et al. measured the cervical consistency index (CCI) using ultrasonography on a cohort of 1031 patients. A correlation was found between the CCI and GA, as well as a sensitivity for prediction of preterm birth [4]. However, in none of these studies, absolute values of stiffness could be retrieved. The use of elastography techniques could only be conclusive with a standardization of the values [5].

Absolute values of cervical stiffness are therefore necessary for an objective assessment of the cervical mechanical state, and the subsequent diagnosis of pre-term labor. This goal can be achieved by using dynamic elastography techniques. Dynamic elastography uses propagation of shear waves within the medium. Some work was conducted in vitro, using Shear Wave Sound Speed (SWS) techniques suggesting that these measurements are sensitive enough to detect differences 
between the nonpregnant nulliparous and multiparous cervix, [6]).

In this paper, we present the Supersonic Shear Imaging (SSI) technique which has the features allowing such an objective and quantitative characterization of tissue stiffness in vivo for applications in obstetrics. The feasibility and reproducibility of such a technique for the assessment of cervical elasticity in vivo in pregnant patients is demonstrated.

The cervical stiffness was quantified in 163 pregnant patients. 70 of these patients were diagnosed at risk of preterm delivery. We demonstrated the inter and intra-operator reproducibility of the technique. We studied the influence of GA on the stiffness of the cervix using the SSI technique. We also evaluated the potential of the SSI technique for the diagnosis of pre-term labour.

\section{MATERIALS AND METHODS}

The Supersonic Shear Imaging (SSI) technique is based on the ultrafast ultrasonic imaging (up to 20000 frames/s) of shear waves generated by an ultrasonic radiation force [10]. Imaging its propagation through the tissue at such ultrafast frame rates enables the measurement of the shear wave speed $c_{T}$. This technique has been thoroughly used for various applications, in various organs such as breast cancer [7] or liver fibrosis [8-9]. An ultrafast scanner (Aixplorer $^{\mathrm{TM}}$, SuperSonic Imagine, Aixen-Provence, France) was used to drive a $7 \mathrm{MHz}$ conventional endocavitary ultrasonic probe (SE 12-3, Supersonic Imagine, Aix-en-Provence, France) allowing classical B-mode acquisition and elasticity maping of the cervix. The Young's modulus $(E)$ was deduced from the shear wave speed $\left(c_{T}\right)$ by:

$$
E \approx 3 \rho c_{T}^{2},
$$

where $\rho$ is the density. This technique allowed elasticity mapping of the whole cervical area. A great advantage of this technique is that it doesn't require additional set-up, or particular training from the physician, and can be performed during routine medical exam.

The Aixplorer $^{\mathrm{TM}}$ ultrasound scanner is FDA (Food and Drugs Administration) approved and received $C E$ clearances for in vivo applications. During the whole study, thermal index (TI) and mechanical index (MI) were kept lower than the FDA thresholds. During ultrasound acquisition, ("Bmode” imaging), values of $\mathrm{TI}$ and $\mathrm{MI}$ were 0.2 and 1.4 respectively. In elastography mode, TI and MI where 0.4 and 1.6 respectively. The total duration of the stiffness measurement using SSI was around a few minutes.

The stiffness quantification was conducted in the lower anterior part of the cervix, within an 8mm diameter's region of interest, using a $7 \mathrm{MHz}$ endocavitary probe. The Shear Wave Elastoraphy mode of the Aixplorer ${ }^{\mathrm{TM}}$ scanner displays both conventional B-mode and stiffness images on the same screen. This allowed an objective and reproducible positioning of the region of interest for stiffness quantification.

The same ROI (8 mm diameter circle) was chosen for all patients. Within the ROI, variations of stiffness were used for an objective criterion for rejection. All patients for which the standard deviation of stiffness values within the ROI was higher than $30 \%$ of the median stiffness value in the ROI were rejected. This corresponds to a total of 33 patients (20\%), among the 163 initally enrolled. All rejections were due to artifacts in the stiffness images.

163 patients were enrolled for this study (age 19-45, mean $31.2+/-5.4$ ), with GA varying from 11.6 to 40 weeks. Among them, 72 were diagnosed at risk of pre-term labour because of contractions and a softened or shortened cervix diagnosed using digital examination and conventional endocavitary ultrasound. All pre-term labour patients were hospitalized. According to the hospital guidelines, pre-term labour diagnosis can only occur between 24 and 36 weeks of GA. Therefore, the pre-term labour patients do not cover the whole range of GA in the studied population. The elastographic images were acquired on patients that had already scheduled a conventional endocavitary ultrasound exam so that it did not represent and additional imaging procedure. All measurements were performed by a trained sonographer.

All stiffness measurements correspond to the mean value obtained on three elastographic images, each of them obtained by repositioning the probe and the ROI on the lower anterior part of the cervix. The inter-operator reproducibility was assessed on three patients by having two practitioners repeat the stiffness measurement with the same procedure: by each practitioner and for each patient, three images were acquired, the ROI was repositioned every time, and the stiffness and errobars were obtained with the median and standard deviation of these three values respectively.

\section{RESULTS}

\section{A. Stiffness monitoring throughout the pregnancy}

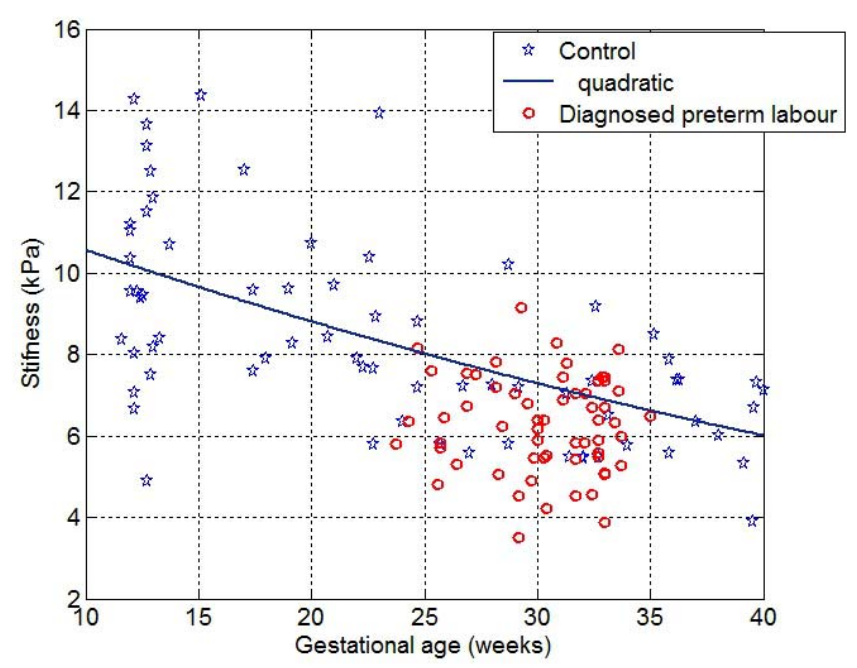

Fig1: Evolution of the cervix stiffness throughout the pregnancy for control (pentagrams) and pre-term labour (circles). Both the average stiffness and the width of the dynamic range for stiffness values significantly decrease with gestational age. Solid line: quadratic fit of the stiffness versus GA for control patients. A majority of the pre-term labour stiffnesses are under this curve.

Fig.1 shows the evolution of cervical stiffness throughout the pregnancy for control (pentagrams) and pre-term labour patients (circles). First, we focus on control patients. A significant trend can be observed: cervical stiffness measured with SSI decreases with the gestational age. It also can be observed that the range of values is larger for earlier terms than for the end of the pregnancy. In the first trimester (GA smaller than 14 weeks), the observed stiffness values was around 9.9 $\mathrm{kPa}+/-2.4 \mathrm{kPa}$. In the second trimester (between 14 and 27 
weeks), the mean value for control patients was $8.9 \mathrm{kPa}+/-$ $3 \mathrm{kPa}$. In the third trimester (later than 27 weeks of GA), the stiffness dropped, as well as the dynamic range for stiffness values: $6.7 \mathrm{kPa}+/-4 \mathrm{kPa}$. A statistically significant difference could be observed between $1^{\text {st }}$ and $3^{\text {rd }}$ trimester distributions ( $p$ $<1 \mathrm{e}^{-6}$ ), as well as between $2^{\mathrm{d}}$ and $3^{\text {rd }}$ trimester distributions ( $\mathrm{p}=$ $1 \mathrm{e}^{-4}$ ). No statistically significant difference could be observed between $1^{\text {st }}$ and $2^{\mathrm{d}}$ trimester stiffness value distributions.

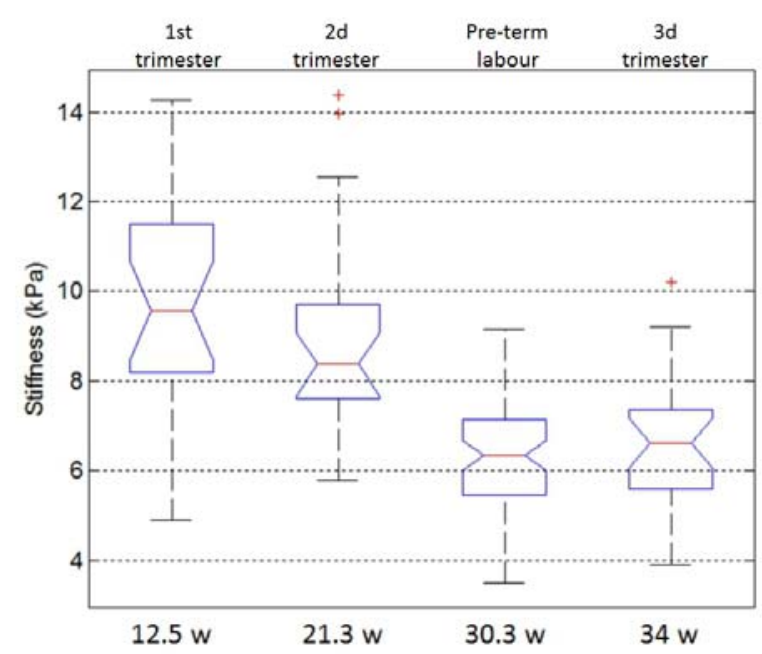

Fig2. Cervical stiffness measured for control patients in the first $(n=22)$, second $(n=22)$ and third trimester $(n=26)$ patients, as well as for patients at risk of pre-term delivery $(n=60)$.

For patients with a diagnosis of preterm labour, stiffness values were measured between 24 and 35 weeks of gestational age. They were globally found around $6.2 \mathrm{kPa}+/-1.2 \mathrm{kPa}$. For matched gestational ages (between 24 and 35 weeks) a statistically significant difference could be observed between the stiffnesses of the control and preterm labour groups $(p=0.01)$. A quadratic fit could be obtained for the stiffness versus gestational age. The mean distance of the pre-term labour data points to this curve was $-1 \mathrm{kPa}$.

\section{B. Pre-term labour}

On Fig. 2, the distributions are presented in the form of boxplots, for each trimester of pregnancy, and for the diagnosed pre-term labour patients. For the latter group, the stiffness values are significantly lower than the values measured in normal patients in the second trimester $(p=1.5 \mathrm{e}-$ 09). The stiffness values of the diagnosed preterm labour group are closer to the values obtained in patients that will soon give birth, in the third trimester of pregnancy (Fig4). In fact, no stastistically significant difference could be observed in these last two groups.

\section{Reproducibility}

Fig3 shows an example of inter-operator reproducibility measurements for the elastographic acquisitions. The measurements presented here were obtained for a third trimester patient (gestional age 32 weeks). The stiffness measurements performed by both practitioners are similar: 10.4 $\pm 1.6 \mathrm{kPa}$ and $11.3 \pm 1 \mathrm{kPa}$. The inter-operator reproducibility of the technique for cervical examination was validated on 3 patients at different stages of pregnancy.

\section{DISCUSSION AND CONCLUSION}

This study demonstrates the feasibility and reproducibility of the Supersonic Shear Imaging technique for cervical stiffness quantification in vivo in pregnant patients. It is, to our knowledge, the first application of transient Shear Wave Elastography to obstetrics.

We have quantified a consistently decreasing cervical stiffness throughout pregnancy, and a much narrower dynamic range of observed cervical stiffness values for later gestational ages.

A significant difference in the stiffness values measured with SSI was observed in the group of patients diagnosed with preterm labour. We observed that cervical stiffness was reduced in patients diagnosed with preterm labor and was similar to the stiffness for control patients close to delivery.

In the current diagnosis of pre-term labour, the only quantitative and objective parameter is the cervical length measured by conventional endocavitary ultrasound.A nonlinear relationship was observed between this parameter and cervical stiffness measured using SSI. In short, long cervices can be soft, and cervical length alone can not be used as a predictive parameter for pre-term labour detection. This is in agreement with the findings by Cabrol et al, reinforcing the idea that these parameters are independent, although modifications of both occur just before labour.

The main limitation of this study is also its main motivation: it relies in the use of an unreliable gold standard for the characterization of pre-term labour. As mentioned above, the digital examination for the assessment of cervical stiffness is highly subjective and not quantitative. Actually, at the maternity hospital were the study was conducted, about half of the pre-term labour patients will eventually carry on with their pregnancy until full term (after 37 weeks of gestational age). In this study, out of the 31 patients who were diagnosed as pre-term labour and who delivered to date, 24 eventually delivered after 37 weeks of pregnancy. It is therefore difficult to know the exact proportion of the patients who were actually in pre-term labour. However, in this study, all the patients who delivered earlier than 36 weeks of pregnancy presented significantly softer cervices at mid pregnancy (Fig.4). This issue only emphasizes the need of an objective and quantitaive tool for the diagnosis of pre-term labour, as there is an issue of over treatement due to poor diagnosis quality.

The second limitation of this study is the fact that the elastographic images were acquired during routine ultrasound exams, usually around 12 weeks of GA, between 20 and 22 weeks of GA, betwen 32 and 34 weeks of GA, and towards the very end of the pregnancy ( 37 weeks of GA and later). Outside these scheduled ultrasound exams, access to healthy patients is difficult in the context of routine pregnancy follow up.

To summarize, the use of SSI for the assessment of cervical stiffness in pregnant paients is feasible and reproducible. We were able to observe a decrease of stiffness with GA, as well as a significant difference between control patients and patients diagnosed at risk of pre-term labour. This method could potentially be used to increase the specificity of the diagnosis, and to identify the patients at risk of pre-term delivery. This technique could be used in the context of routine conventional endocavitary ultrasound exams. It also has a great potential for the prediction of the success of labour induction 


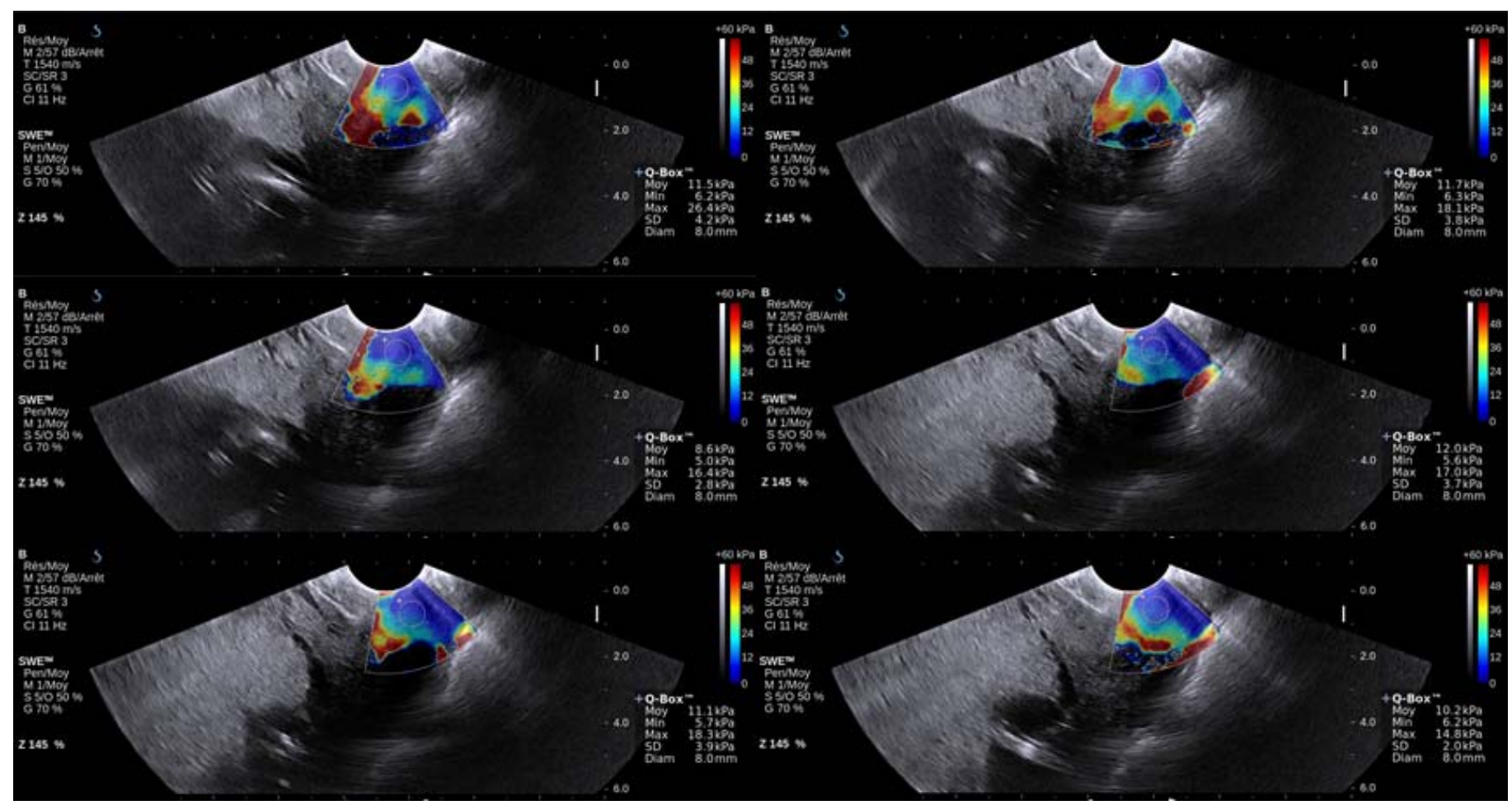

Fig3. Elastographic and Bmode images of the cervix of a pregnant patient in the first trimester (32 weeks of GA) taken by 2 practitioners. Left: First practitioner:10.4 kPa $\pm 1.6 \mathrm{kPa}$. Right: Second practitioner:11.3 $\mathrm{kPa} \pm 1 \mathrm{kPa}$.

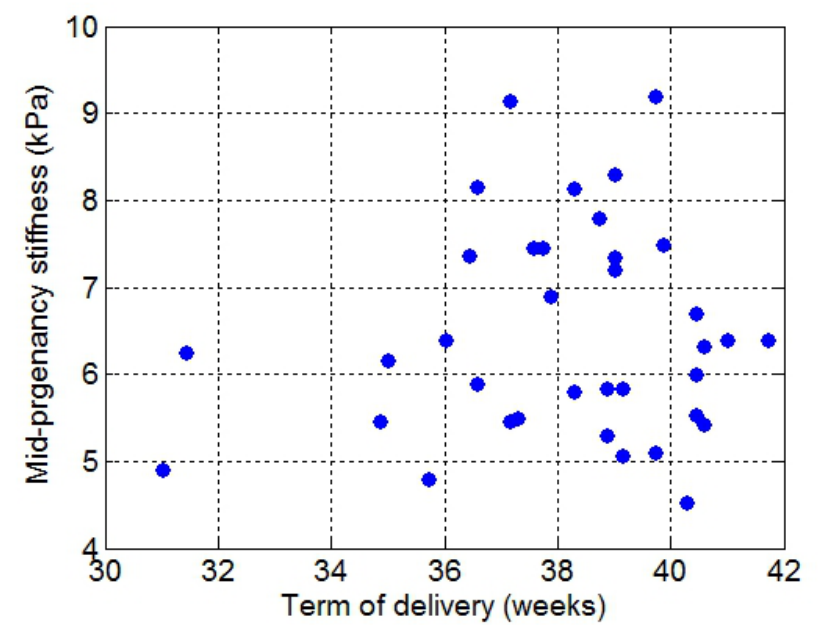

Fig4. Cervical stiffness measured at mid-pregnancy (between 24 and 35 weeks) versus term at delivery. All the patients who delivered early (before 36 weeks of GA) exhibit a much softer cervix.

\section{ACKNOWLEDGMENT}

This work is supported by LABEX WIFI (Laboratory of Excellence within the French Program "Investments for the Future") under references ANR-10-LABX-24 and ANR-10-IDEX-0001-02 PSL*. of premature delivery in patients with preterm labor and intact membranes, Am. J. Obstet. Gyn. 171(4), 1994

[2] Cabrol D, et al Prognostic value of cervical distensibility index measurement in the outcome of pregnancies with threatened premature labor, Gynecol Obstet Invest, 32(1), 28-32, 1991

[3] Molina FS, et al Quantification of cervical elastography : a reproducibility study. Ultrasound Obstet. Gynecol 39, 685-689, 2012

[4] Bauer M. et al, Assessment of the in vivo biomechanical properties of the human uterine cervix in pregnancy using the aspiration test: A feasibility study, Eur J Obstet Gynecol Reprod Biol, 144S:S77:81, 2009

[5] Feltovitch $\mathrm{H}$, et al Beyond cervical length: emerging technologies for assessing the pregnant cervix, Am. J. Obstet. Gyn, 207(5), 345-54,2012

[6] Reusch LM, et al, Comparison of Ultrasonic Measurements of Nulliparous versus Multiparous Cervices, IEEE Ultrason Symp 2011

[7] Tanter M. et al, Quantitative assessment of breast lesion viscoelasticity: initial clinical results using supersonic shear imaging, Ultrasound Med. Biol. 34(9), 1373-86, 2008

[8] Muller M. et al, Quantitative viscoelasticity mapping of human liver using supersonic shear imaging: preliminary in vivo feasibility study, Ultrasound Med. Biol. 35(2), 219-229, 2009

[9] Bavu E. et al, Noninvasive in vivo liver fibrosis evaluation using supersonic shear imaging: a clinical study on 113 hepatitis $\mathrm{C}$ virus patients., Ultrasound Med. Biol., 37(9), 1361-73, 2011

[10] Bercoff J. et al, Supersonic Shear Imaging: A new technique for soft tissue elasticity mapping, IEEE Uffc 51(4), april 2004

\section{REFERENCES}

[1] Gomez R. et al, Ultrasonographic examination of the uterine cervix is better than cervical digital examination as a predictor of the likelihood 